\title{
Towards understanding the Pierce-Birkhoff conjecture via MV-algebras
}

\author{
Serafina Lapenta \\ Department of Mathematics, Computer Science and Economics, \\ University of Basilicata, Viale dell'Ateneo Lucano, 10 Potenza, Italy \\ serafina.lapenta@unibas.it \\ Ioana Leuştean \\ Department of Computer Science, \\ Faculty of Mathematics and Computer Science, University of Bucharest, \\ Academiei nr.14, sector 1, C.P. 010014, Bucharest, Romania \\ ioana@fmi.unibuc.ro
}

\begin{abstract}
Our main issue was to understand the connection between Łukasiewicz logic with product and the Pierce-Birkhoff conjecture, and to express it in a mathematical way. To do this we define the class of $f \mathrm{MV}$-algebras, which are MV-algebras endowed with both an internal binary product and a scalar product with scalars from $[0,1]$. The proper quasi-variety generated by $[0,1]$, with both products interpreted as the real product, provides the desired framework: the normal form theorem of its corresponding logical system can be seen as a local version of the Pierce-Birkhoff conjecture.
\end{abstract}

\section{Introduction}

Lukasiewicz $\infty$-valued logic with the primitive connectives $\rightarrow$ and $\neg$ has as set of truth values the real interval $[0,1]$ with $x \rightarrow y=\max (0,1-x+y)$ and $\neg x=1-x$ for any $x, y \in[0,1]$. The completeness theorem states that a formula is provable if and only if it holds in the standard model $[0,1]$. The corresponding algebraic structures, called $M V$-algebras [4], were defined as structures $\left(A, \oplus,{ }^{*}, 0\right)$ of type $(2,1,0)$ satisfying some appropriate axioms. The variety of MV-algebras is generated by $\left([0,1], \oplus,{ }^{*}, 0\right)$ where $x \oplus y=\min (1, x+y)$ and $x^{*}=1-x$ for any $x, y \in[0,1]$.

One major result in the theory of $\mathrm{MV}$-algebras is the categorical equivalence with the Abelian lattice-ordered groups with strong unit [24]. As a consequence, for any MV-algebra $A$ there exists an Abelian lattice-ordered group with strong unit $(G, u)$ such that $A \simeq[0, u]_{G}$, where $\left[0, u_{G}\right]=\left([0, u], \oplus,^{*}, 0\right)$ with $x \oplus y=$ $u \wedge(x+y)$ and $x^{*}=u-x$ for any $x, y \in[0, u]$. 
The standard MV-algebra $[0,1]$ is closed with respect to the real product, so the study of the structure obtained by endowing the MV-algebras with a product operation was an active research direction. Our model in this case is $\left([0,1], \oplus, \cdot{ }^{*}, 0\right)$, where $\left([0,1], \oplus,{ }^{*}, 0\right)$ is the standard MV-algebra and $\cdot$ is the real product. We shall only mention the references strictly related with our development, but the literature on the subject is far richer. For such structures further categorical equivalences with classes of lattice-ordered structures were proved.

If we consider the real product as a binary operation on $[0,1]$, the corresponding structures are MV-algebras $A$ endowed with an operation $\cdot: A \times A \rightarrow A$. These were introduced and studied in [8] under the name of $P M V$-algebras and they are categorically equivalent to a class of lattice-ordered rings with strong unit. Particular important subclasses were further studied in [21, 22]. As proved in [21, the variety of (commutative and unital) PMV-algebras is larger than the variety generated by $\left([0,1], \oplus, \cdot,{ }^{*}, 0\right)$. The quasi-variety $\operatorname{ISP}([0,1])$ was characterized in 22].

One can also consider the real product on $[0,1]$ as a scalar multiplication. Our standard model in this case is $\left([0,1], \oplus,{ }^{*},\{\alpha \mid \alpha \in[0,1]\}, 0\right)$ where $x \mapsto \alpha x$ is a unary operation for any $\alpha \in[0,1]$. These structures are investigated in [9] under the name of Riesz $M V$-algebras and they are categorically equivalent to Riesz spaces (vector lattices) with strong unit. The variety of Riesz MV-algebras is generated by the standard model $[0,1]$.

Both for PMV-algebras and Riesz MV-algebras logical systems were developed [13, 9] and such systems are conservative extensions of Łukasiewicz logic. One of the main theorems of Łukasiewicz logic states that the term functions corresponding to the formulas of Eukasiewicz logic with $n$ variables $(n \geq 1)$ are exactly the continuous $[0,1]$-valued piecewise linear functions with integer coefficients defined on $[0,1]^{n}[20$. This can be seen as a normal form theorem for Eukasiewicz logic. A similar result was proved in [9] for the logical system that has Riesz MV-algebras as models; in this case the piecewise linear functions have real coefficients. In [23, Introduction] it is stated that a similar result for PMV-algebras is related to the Pierce-Birkhoff conjecture [3] (see Section 5] for explicit formulations).

Our main issue was to understand the connection between Łukasiewicz logic with product (in a broad sense) and the Pierce-Birkhoff conjecture, and to express it in a mathematical way. To do this, we study the class of structures obtained by endowing MV-algebras with both the internal binary product and the scalar product (as a family of unary operations). We called $f M V$-algebras the structures obtained in this way and the model we focus on is $\mathbf{I}=\left([0,1], \oplus, \cdot{ }^{*},\{\alpha \mid \alpha \in[0,1]\}, 0\right)$.

We can briefly express our final conclusions as follows:

(1) the variety of $f \mathrm{MV}$-algebras is larger then $\operatorname{HSP}(\mathbf{I})$;

(2) we characterize $\operatorname{ISP}(\mathbf{I})$; we called $F R^{+}$-algebras the members of $\operatorname{ISP}(\mathbf{I})$;

(3) the logical system $\mathcal{F} \mathcal{M V} \mathcal{L}^{+}$that has $\mathrm{FR}^{+}$-algebras as models is a conservative extension of Łukasiewicz logic and it is complete w.r.t. I;

(4) the normal form theorem for $\mathcal{F} \mathcal{M V} \mathcal{L}^{+}$(Conjecture 5.1) is, in our approach, 
the link between the Pierce-Birkhoff conjecture and Lukasiewicz logic with product. We proved it for $n \leq 2$, due to the fact that the Pierce-Birkhoff conjecture is proved in this case [19]. Note that Conjecture 5.1]does not immediately imply, nor it is implied by the Pierce-Birkhoff conjecture, additional results are needed and, in our opinion, these results belong more to the area of algebraic geometry than to the area of logic.

Note that for $\mathrm{FR}^{+}$-algebras we prove the subdirect representation w.r.t. totally-ordered structures and a representation as algebras of * $[0,1]$-valued functions, where $*[0,1]$ is an ultrapower of $[0,1]$.

Section 1 contains the preliminary notions. In Section 2 we define the $f \mathrm{MV}$ algebras, we prove a subdirect representation theorem and the categorical equivalence between $f \mathrm{MV}$-algebras and a subclass of $f$-algebras. In Section 3 we investigate special classes of $f \mathrm{MV}$-algebras and in the last subsection we introduce the class of $\mathrm{FR}^{+}$-algebras. Logical systems are developed in Section 4 . In Section 5 we relate previous results with the Pierce-Birkhoff conjecture.

\section{Preliminaries}

We recall the algebraic structures involved in our development.

\section{$1.1 \quad \mathrm{MV}$-algebras and $\ell u$-groups}

An $M V$-algebra is an algebraic structure $\left(A, \oplus,{ }^{*}, 0\right)$ such that $(A, \oplus, 0)$ is an abelian monoid, $\left(x^{*}\right)^{*}=x$ and $\left(x^{*} \oplus y\right)^{*} \oplus y=\left(y^{*} \oplus x\right)^{*} \oplus x$ for any $x, y \in A$. If $A$ is an MV-algebra, one can define $x \odot y=\left(x^{*} \oplus y^{*}\right)^{*}, x \ominus y=x \odot y^{*}$, $x \rightarrow y=x^{*} \oplus y$ and $1=0^{*}$, for any $x, y \in A$. The order defined by setting $x \leq y$ if and only if $x \odot y^{*}=0$ is a lattice order such that $x \vee y=\left(x \odot y^{*}\right) \oplus y$ and $x \wedge y=\left(x^{*} \vee y^{*}\right)^{*}=x \odot\left(x^{*} \oplus y\right)$ for any $x, y \in A$. We refer to [6] for all the unexplained notions concerning MV-algebras and to [25] for advanced topics.

The variety of MV-algebras is generated by $\left([0,1], \oplus,{ }^{*}, 0\right)$ where $x \oplus y=$ $\min (1, x+y)$ and $x^{*}=1-x$ for any $x, y \in[0,1]$. Any MV-algebra is a subdirect product of totally-ordered MV-algebras. Moreover, any MV-algebra can be embedded into a subalgebra of an ultrapower of $[0,1]$.

An ideal $I$ in an MV-algebra $A$ is a nonempty subset such that for any $x, y \in I, x \oplus y \in I$ and if $x \leq y$ with $y \in I$ then $x \in I$. The intersection of all maximals ideals in an MV-algebra is called radical, and it is denoted by $\operatorname{Rad}(A)$. An MV-algebra is called semisimple if $\operatorname{Rad}(A)=\{0\}$. Recall that any semisimple MV-algebra is isomorphic to a subalgebra of the MV-algebra $C(X)$ of $[0,1]$-valued continuous functions defined on a suitable topological space $X$.

The relation between MV-algebras and lattice-ordered groups plays a crucial role in our approach. A lattice-ordered group is a group is a structure $(G,+, 0, \leq)$ such that $(G,+, 0)$ is a group, $(G, \leq)$ is a lattice and $x \leq y$ implies $x+z \leq y+z$ for any $x, y, z \in G[1,2]$. A strong unit $u$ is an element $u>0$ such that for any $x \in G$ there exists an $n \in \mathbb{N}$ with $x \leq n u$. An lu-group is a pair $(G, u)$ where $G$ is an Abelian lattice-ordered group and $u$ is a strong unit in $G$. For any element $u \in G, u>0$ we define the unit interval as 
$[0, u]_{G}=\left([0, u]=\{x \in G \mid 0 \leq x \leq u\}, \oplus,{ }^{*}, 0\right)$, where

$x \oplus y=u \wedge(x+y)$ and $x^{*}=u-x$ for any $x, y \in[0, u]$. The structure $[0, u]_{G}$ is an MV-algebra.

An $\ell$-group $G$ is said to be Archimedean if $n x \leq y$ for any $n \in \mathbb{N}$, with $x, y \in G$, implies $x \leq 0$.

Let $\mathbf{M V}$ denote the category of MV-algebras and auG the category whose objects are $\ell u$-groups and whose morphisms are homomorphisms of $\ell u$-groups that preserve the strong unit.

Theorem 1.1. 24] Let $\Gamma$ be the functor defined by $\Gamma: \mathbf{a u G} \rightarrow \mathbf{M V} \quad \Gamma(G, u)=[0, u]_{G}$ and $\Gamma(h)=\left.h\right|_{[0, u]_{G}}$,

then $\Gamma$ establishes a categorical equivalence. Moreover, an $M V$-algebra is semisimple if and only if the corresponding lu-group is Archimedean.

\section{2 $\quad \mathrm{PMV}$-algebras and $\ell u$-rings}

A PMV-algebra [8] is a structure $\left(P, \oplus,{ }^{*}, \cdot, 0\right)$ such that $\left(P, \oplus,{ }^{*}, 0\right)$ is an MValgebra and the binary operation - satisfies the following, for any $x, y, z \in P$ :

$(\mathrm{PMV} 1) z \cdot\left(x \odot(x \wedge y)^{*}\right)=(z \cdot x) \odot(z \cdot(x \wedge y))^{*}$

$(\mathrm{PMV})\left(x \odot(x \wedge y)^{*}\right) \cdot z=(x \cdot z) \odot((x \wedge y) \cdot z)^{*}$.

$(\mathrm{PMV} 3) x \cdot(y \cdot z)=(x \cdot y) \cdot z$.

A PMV-algebra that has unit for product is called unital, and in this case the unit is $1=0^{*}$ [8, Proposition 3.1].

A - -ideal $I$ in a PMV-algebra $P$ is an MV-ideal that satisfies the condition $x \cdot y \in I$ and $y \cdot x \in I$ for any $x \in I$ and $y \in P$.

A PMVf-algebra is an PMV-algebra that satisfies the following condition:

(f) $x \wedge y=0$ implies $(x \cdot z) \wedge y=(z \cdot x) \wedge y=0$ for any $x, y, z \in P$.

By [8, Theorem 5.4] this condition has an equational form. Trivially, unital PMV-algebras are PMVf-algebras and the standard MV-algebra [0,1] endowed with the usual real product is a PMV $f$-algebra. Any $\operatorname{PMV} f$-algebra is a subdirect product of totally-ordered $\mathrm{PMV} f$-algebra.

The Product MV-algebras defined in [21, Definition 2.8] are the unital and commutative PMV-algebras from [8] (21], [22, Theorem 2.8]). A $P M V^{+}$algebra $P$ is a unital and commutative PMV-algebra that satisfies the quasiidentity $\left(x^{2}=0 \Rightarrow x=0\right)$ for any $x \in P[22]$. Any $P M V^{+}$-algebra is a subdirect product of totally-ordered $P M V^{+}$-algebras [13].

Theorem 1.2. [22] The class of all $P M V^{+}$-algebras is the quasi-variety generated by $\left([0,1], \oplus, \cdot{ }^{*}, 0\right)$. Moreover, any totally-ordered $P M V^{+}$-algebra embeds into a subalgebra of an ultrapower of $[0,1]$.

An $\ell$-ring $R$ is an $\ell u$-group endowed with an operation $\cdot: R \times R \rightarrow R$ such that $(R, \cdot)$ is a ring and $x \cdot y \geq 0$ whenever $x, y \geq 0[1,3]$. An $f$-ring is a lattice-ordered ring $R$ such that $x \wedge y=0$ implies $(x \cdot z) \wedge y=(z \cdot x) \wedge y=0$, for any $z \geq 0$ and $x, y \in R$.

Denoted by PMV the category of PMV-algebras and product preserving homomorphisms of MV-algebras and by $\mathbf{u R}$ the category of $\ell u$-rings and 
homomorphisms of $\ell u$-rings, we extend the functor $\Gamma$ and we get a functor $\Gamma_{(\cdot)}: \mathbf{u R} \rightarrow \mathbf{P M V}$, with $\Gamma_{(\cdot)}(R, u)=[0, u]_{R}$ and $\Gamma_{(\cdot)}(h)=\left.h\right|_{[0, u]_{R}}$, where $(R, u)$ is an object and $h$ is a morphism in $\mathbf{u R}$. The functor $\Gamma_{(\cdot)}$ establishes a categorical equivalence [8]. Moreover, $f$-rings correspond to $\operatorname{PMV} f$-algebras.

\subsection{Riesz MV-algebras and Riesz spaces}

A Riesz MV-algebra 9] is a structure $\left(R, \oplus,{ }^{*},\{\alpha \mid \alpha \in[0,1]\}, 0\right)$ such that $\left(R, \oplus,{ }^{*}, 0\right)$ is an MV-algebra and $\{\alpha \mid \alpha \in[0,1]\}$ is a family of unary operations satisfying the following identities for any $\alpha, \beta \in[0,1]$ and any $x, y \in R$ :

$(\mathrm{RMV1}) \alpha\left(x \odot y^{*}\right)=(\alpha x) \odot(\alpha y)^{*}$

$(\mathrm{RMV} 2) \max (0, \alpha-\beta) x=(\alpha x) \odot(\beta x)^{*}$,

$(\mathrm{RMV} 3) \alpha(\beta x)=(\alpha \beta) x$,

(RMV4) $1 x=x$.

By [9, Corollary 2] any homomorphism of MV-algebras between Riesz MValgebras preserves the additional unary operations, so it is a homomorphism of Riesz MV-algebras. Similarly, if $R$ is a Riesz MV-algebra then any ideal $I$ of the MV-algebra reduct is closed to the unary operations: $\alpha x \in I$ for any $x \in I$ and $\alpha \in[0,1]$. The variety of Riesz MV-algebras is generated by $\left([0,1], \oplus, \cdot,^{*},\{\alpha \mid \alpha \in[0,1]\}, 0\right)[9]$.

A Riesz space is a lattice ordered linear space over the real field, that is an $\ell$-group endowed with a scalar product with scalars over $\mathbb{R}$ such that $\alpha x \geq 0$ for any $\alpha$ and $x$ non-negative [26]. When we deal with $\ell u$-groups, we obtain a Riesz space with strong unit.

Let uRS be the category of Riesz spaces with strong unit and linear homomorphisms of $\ell u$-groups, and let RMV be the category of Riesz MV-algebras with homomorphisms of Riesz MV-algebras. We get a functor $\Gamma_{\mathbb{R}}: \mathbf{u R S} \rightarrow$ $\mathbf{R M V}$, with $\Gamma_{\mathbb{R}}(R, u)=[0, u]_{R}$ and $\Gamma_{\mathbb{R}}(h)=\left.h\right|_{[0, u]_{R}}$, where $(R, u)$ is an object and $h$ is a morphism in uRS. The functor $\Gamma_{\mathbb{R}}$ establishes a categorical equivalence 9 .

Putting all together, we get the following commutative diagram

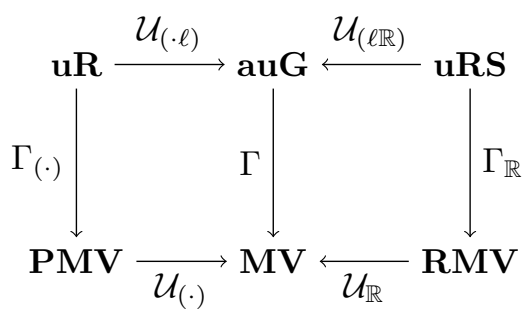

Figure 1.

where $\mathcal{U}_{\mathbb{R}}, \mathcal{U}_{(\ell \mathbb{R})}, \mathcal{U}_{(\cdot)}, \mathcal{U}_{(\cdot \ell)}$ are forgetful functors. 


\section{$1.4 f$-algebras}

An $f$-algebra is a strucure $(L,+, \cdot,\{r \mid r \in \mathbb{R}\}, 0, \leq)$ such that $(L,+, \cdot, 0, \leq)$ is a $f$-ring, $(L,+,\{r \mid r \in \mathbb{R}\}, 0, \leq)$ is a Riesz space and the following condition is satisfied:

(fa) $r(x \cdot y)=(r x) \cdot y=x \cdot(r y)$ for any $x, y \in L$ and any $r \in \mathbb{R}$.

If there exists an element $e$ such that $x e=e x=x$ for any $x \in L, L$ will be called unital, and $e$ is the unit element for $L$. An $f$-algebra with strong unit is an $f$-algebra in which the underlying Riesz space is a Riesz space with strong unit. This notion was first introduced in [3], for further details see also [1, 12, 26].

Definition 1.1. In the sequel, an fu-algebra is a pair $(V, u)$, where $V$ is an $f$-algebra and $u \in V$ is a strong unit such that $u \cdot u \leq u$. If $\left(V_{1}, u_{1}\right)$ and $\left(V_{2}, u_{2}\right)$ are $f u$-algebras then $g: V_{1} \rightarrow V_{2}$ is a homomorphism of $f u$-algebras if it is a homomorphism of $f$-algebras and $g\left(u_{1}\right)=u_{2}$.

Note that, for an $f u$-algebra $(V, u)$, the interval $[0, u]$ is closed with respect to the product operations and with respect to the scalar multiplication by elements from $[0,1]$.

\section{$2 \quad f \mathrm{MV}$-algebras}

\subsection{Definitions and first results}

In the following we define the structure of $f M V$-algebra, an appropriate notion of an ideal and we prove a subdirect representation theorem.

Definition 2.1. An fMV-algebra is a structure $\left(A, \oplus, \cdot{ }^{*},\{\alpha \mid \alpha \in[0,1]\}, 0\right)$ which satisfies the following properties for any $x, y, z \in A$ and $\alpha \in[0,1]$ :

$(f \mathrm{MV} 1) \quad\left(A, \oplus, \cdot{ }^{*}, 0\right)$ is a PMV $f$-algebra,

$(f \mathrm{MV} 2) \quad\left(A, \oplus,{ }^{*},\{\alpha \mid \alpha \in[0,1]\}, 0\right)$ is a Riesz MV-algebra,

$(f \mathrm{MV} 3) \quad \alpha(x \cdot y)=(\alpha x) \cdot y=x \cdot(\alpha y)$.

We say that the $f \mathrm{MV}$-algebra $A$ is unital (commutative) if its PMV-algebra reduct $\left(A, \oplus, \cdot,{ }^{*}, 0\right)$ is unital (commutative).

Remark 2.1. The class of $f M V$-algebras is a variety, denoted by $\mathbb{F M V}$.

One can see that an $f \mathrm{MV}$-algebra has an MV-algebra reduct, a Riesz MValgebra reduct, as well as a PMV-algebra reduct and the forgetful functors are summarized in the following diagram:

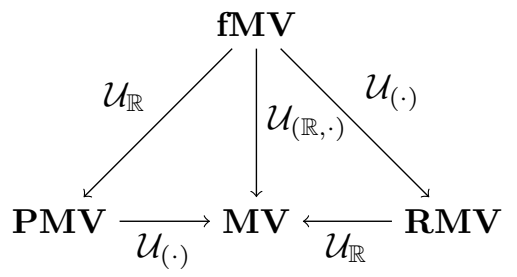

Figure 2 . 
Lemma 2.1. If an $f M V$-algebra $A$ is unital then the unit is 1 .

Proof. If $A$ is a unital $f \mathrm{MV}$-algebra, then $\mathcal{U}_{\mathbb{R}}(A)$ is a unital PMV-algebra. The result follows from [8, Proposition 3.1].

The following examples play an important role in our development.

Example 2.1. 1. The real interval $[0,1]$ with the standard structure of MValgebra is an $f \mathrm{MV}$-algebra where both the scalar multiplication and the internal product coincides with the real product. We always consider $[0,1]$ endowed with this structure, but we note that it does not generate the variety of all $f \mathrm{MV}$-algebras, but only a proper subclass (see Section 3.3).

2. If $(V, u)$ is an $f u$-algebra then, by [9, Lemma 5] and [8, Theorem 5.2], the interval $[0, u]$ is trivially a Riesz MV-algebra and a PMVf-algebra. The condition $(f \mathrm{MV} 3)$ from Definition 2.1 holds, since it is already satisfied in $(V, u)$. Hence $[0, u]_{V}=\left([0, u], \oplus, \cdot{ }^{*},\{\alpha \mid \alpha \in[0,1]\}, 0\right)$ is an $f$ MV-algebra.

We list some further examples.

Example 2.2. 1. For any nonempty set $X,[0,1]^{X}$ with point-wise operations of MV-algebra and point-wise products is an $f \mathrm{MV}$-algebra.

2. If $(X, \tau)$ is a topological compact and Hausdorff space then $C(X)=\{f$ : $X \rightarrow[0,1] \mid f$ continuous $\}$ with point-wise operations of MV-algebra and point-wise products is an $f \mathrm{MV}$-algebra.

3. On the Riesz MV-algebra $[0,1]^{2}$ with coordinate-wise operations of MValgebra and coordinate-wise scalar product, we define the internal product by $\left(x_{1}, y_{1}\right) \cdot\left(x_{2}, y_{2}\right)=\left(x_{1} x_{2}, 0\right)$ for any $x_{1}, x_{2}, y_{1}, y_{2} \in[0,1]$. One can easily prove that the structure obtained in this way is an $f \mathrm{MV}$-algebra.

4. Let $H$ be an one dimensional Hilbert space over the complex field, $\mathcal{H}$ the set of all bounded Hermitian operators on $H$ and assume $\mathcal{D}$ is a nonempty subset of $\mathcal{H}$. Hence $\mathcal{C}^{\prime \prime}(\mathcal{D})$, the bicommutant of $\mathcal{D}$ is an $f$-algebra with strong unit $E$, where $E$ is the identity operator. For more details we refer to [16, Chapter 8]. Then, the unit interval $[\theta, E]$ is an $f \mathrm{MV}$-algebra, where $\theta$ is the null operator.

In 8 the proper notion of ideal was defined for PMV-algebras, under the name of -ideal. We follow a similar approach for $f \mathrm{MV}$-algebras.

Definition 2.2. If $A$ is an $f \mathrm{MV}$-algebra then a subset $I \subset A$ is an ideal if it is an ideal of the MV-algebra reduct of $A$. An ideal $I$ of $A$ is a -ideal if $l$ if it is a --ideal of the PMV-algebra reduct of $A$.

Remark 2.2. Assume $A$ is an $f \mathrm{MV}$-algebra and $I$ is a --ideal of $A$.

(1) Since $\alpha x \leq 1 x=x$ for any $\alpha \in[0,1]$ and $x \in A$, then $x \in I$ implies $\alpha x \in I$ for any $\alpha \in[0,1]$. 
(2) When $A$ is unital, --ideals coincide with the ideals of the MV-algebra reduct of $A$, since for any $x \in I$ and any $y \in A$ we get $x \cdot y \leq x \cdot 1=x$.

For a -ideal $I$ of an $f \mathrm{MV}$-algebra $A$, we define a congruence relation by:

$$
x \sim_{I} y \quad \text { if and only if } \quad x \odot y^{*} \in I \text { and } y \odot x^{*} \in I
$$

By [9, Remark 2], the quotient of $A$ with respect to $\sim_{I}$ is a Riesz MV-algebra. In order to prove that the quotient is a $\operatorname{PMV} f$-algebra, we need to prove that

$$
\text { if } x \sim_{I} y \text {, then } z \cdot x \sim_{I} z \cdot y \text { and } x \cdot z \sim_{I} y \cdot z .
$$

But this follows trivially by conditions (f1) and (f2) in Section 1.2 We can now define the product in the quotient algebra $A / \sim_{I}$ by

and $A / I$ is an $f \mathrm{MV}$-algebra.

$$
[x] \cdot[y]=[x \cdot y]
$$

We are ready to prove the subdirect representation theorem with respect to totally-ordered structures.

Theorem 2.1. Any $f M V$-algebra $A$ is a subdirect product of totally-ordered $f M V$-algebras.

Proof. By [8, Theorem 5.5], $\mathcal{U}_{\mathbb{R}}(A)$ is a subdirect product of totally-ordered PMV $f$-algebras, i.e. there exists a family $\left\{A_{k}\right\}_{k \in K}$ of totally-ordered PMVfalgebras and a subdirect representation $\iota: A \rightarrow \prod_{k \in K} A_{k}$. Consequently, there is a family $\left\{P_{k}\right\}_{k \in K}$ of prime -ideals of $\mathcal{U}_{\mathbb{R}}(A)$ such that $\cap\left\{P_{k} \mid k \in K\right\}=\{0\}$. By definition, $P_{k}$ is also a $\cdot$-ideal of $A$, so $A_{k}$ is a totally-ordered $f \mathrm{MV}$-algebras for any $k \in K$.

\subsection{Categorical equivalence with $f u$-algebras}

The aim of this section is to prove a categorical equivalence between $f \mathrm{MV}$ algebras and $f$-algebras, generalizing the similar results for MV-algebras, PMValgebra and Riesz MV-algebras.

We denote by $\mathbf{f M V}$ the category whose objects are $f \mathrm{MV}$-algebras and whose morphisms are homomorphisms of $f \mathrm{MV}$-algebras and by fuAlg the category whose objects are $f u$-algebras and whose morphisms are homomorphisms of $f$ algebras that preserve the strong unit.

The functor $\Gamma_{f}:$ fuAlg $\rightarrow \mathbf{f M V}$ is defined by

- $\Gamma_{f}(V, u)=[0, u]_{V}$ as in Example 2.1.

- if $g:\left(V_{1}, u_{1}\right) \rightarrow\left(V_{2}, u_{2}\right)$ is a homomorphism of $f u$-algebras then $\Gamma_{f}(g)$ is defined as $\left.g\right|_{\left[0, u_{1}\right]}$.

Theorem 2.2. The functor $\Gamma_{f}$ establishes a categorical equivalence.

Proof. $\Gamma_{f}$ is trivially well defined. First, we prove that for any $f$ MV-algebra $A$, there exists an fu-algebra $(\mathrm{V}, \mathrm{u})$ such that $A \simeq \Gamma_{f}(V, u)$.

By [9, Proposition 3] there exists a Riesz space with strong unit $(V, u)$ such that $\mathcal{U}_{(\cdot)}(A) \simeq \Gamma_{\mathbb{R}}(V, u)$. Since $A$ has a PMV-algebra reduct, by $[8$, Theorems 4.2 and 5.2], $(V, u)$ can be endowed with an internal product $\cdot: V \times V \rightarrow V$ 
such that $(V, \cdot)$ is an $f$-ring. Moreover, by easy computation $(V, u)$ satisfies the condition (fa).

One can easily see that the functor $\Gamma_{f}$ is faithful: if we have $h_{1}, h_{2}$ : $\left(V_{1}, u_{1}\right) \rightarrow\left(V_{2}, u_{2}\right)$, such that $\Gamma_{f}\left(h_{1}\right)=\Gamma_{f}\left(h_{2}\right)$, then $h_{1}$ and $h_{2}$ coincide on the generating set of $\left(V_{1}, u_{1}\right)$, therefore they coincide on the whole algebra, and $h_{1}=h_{2}$.

To end the proof, we show that $\Gamma_{f}$ is full. Let A, B be $f \mathrm{MV}$-algebras such that $A \simeq \Gamma_{f}\left(V_{1}, u_{1}\right), B \simeq \Gamma_{f}\left(V_{2}, u_{2}\right)$ for $\left(V_{1}, u_{1}\right)$ and $\left(V_{2}, u_{2}\right)$ objects in fuAlg. If $h: A \rightarrow B$ is a homomorphism of $f \mathrm{MV}$-algebras then, in particular, $h$ is a homomorphism of PMV-algebras between the PMV-algebra reducts of $A$ and $B$. By [8, Theorem 4.2], there exists a homomorphism of $\ell u$-rings $h^{\sharp}:\left(V_{1}, u_{1}\right) \rightarrow$ $\left(V_{2}, u_{2}\right)$ extending $h$. By [2, Chapter XV Section 2 Corollary], any morphism of $\ell$-groups between Riesz spaces is linear. It follows that $h^{\sharp}:\left(V_{1}, u_{1}\right) \rightarrow\left(V_{2}, u_{2}\right)$ is also linear, so it is a homomorphism of $f u$-algebras.

We are now able to complete the diagram:

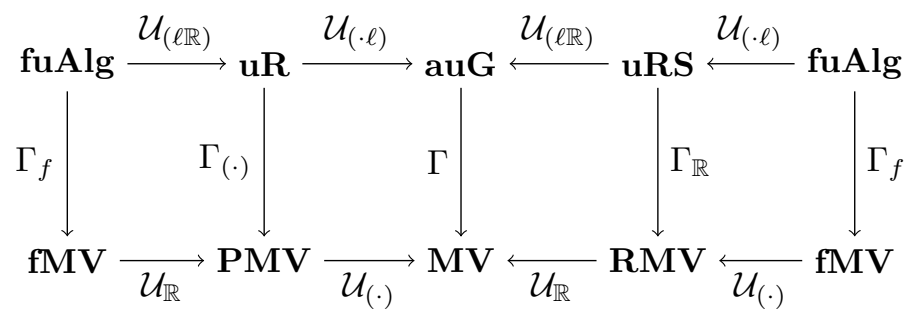

Figure 3.

Corollary 2.1. Let $A \simeq \Gamma_{f}(V, u)$. If $A$ is unital then $(V, u)$ is unital and its unit coincide with the strong unit $u$.

Proof. If follows from Lemma 2.1 and [8, Proposition 3.1 and Theorem 3.3]

Corollary 2.2. Any homomorphism of PMV-algebras between the PMV-algebra reducts of two $f M V$-algebras preserves the scalar product, i.e. it is a morphism of fMV-algebras.

Proof. By [2, Chapter XV Section 2 Corollary] any morphism of $\ell$-groups between Riesz spaces is linear. Then we apply Theorem 2.2

Corollary 2.3. Let $A$ be an $f M V$-algebra in which the internal product is commutative. Then $x \cdot y=(x \wedge y) \cdot(y \vee x)$.

Proof. By Theorem 2.2, there exists an $f u$-algebra $(V, u)$ such that $A \simeq \Gamma_{f}(V, u)$. By [26, Theorem 142.4], $x \cdot y=(x \wedge y) \cdot(y \vee x)$ for any $x, y \in V$.

Let $V$ be an $f$-algebra. Recall that a subset $J$ of $V$ is an $\ell$-ideal if it is a linear subspace that satisfies the following conditions:

$(\ell 1)$ if $x \in V, y \in J,|x| \leq|y|$ then $x \in J$,

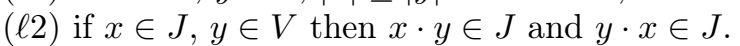


Remark 2.3. Let $(\mathrm{V}, \mathrm{u})$ be an $f u$-algebra, and let $A=\Gamma(V, u)$. We define the maps

$\Phi: I \mapsto \Phi(I)=\{x \in V|| x \mid \wedge u \in I\}$ and $\Psi: H \mapsto \Psi(H)=H \cap[0, u]$

for any -ideal $I$ of $A$ and any $\ell$-ideal $H$ of $V$. Then $\Phi$ and $\Psi$ are orderisomorphisms from the set of -ideals of $A$ to the set of $\ell$-ideals of $(V, u)$. The proof follows by the similar result for PMV-algebras and $\ell$-rings 8 , Theorem $5.1]$ and by the fact that any ideal of $\ell$-ring considered in [8] is an $\ell$-ideal by [2, Chapter XV Section 2 Lemma 1].

Definition 2.3. For a --ideal $I$ of an $f \mathrm{MV}$-algebra $A$, the nil-radical is

$$
\sqrt{I}=\left\{x \in A \mid x^{n} \in I \text { for some } n \in \mathbb{N}\right\},
$$

where $x^{n}$ is $\overbrace{x \cdot \ldots \cdot x}^{n \text { times }}$.

Proposition 2.1. If $I$ is a-ideal in a commutative $f M V$-algebra $A$, then $\sqrt{I}$ is $a \cdot-$ ideal in $A$.

Proof. Let $(V, u)$ be the $f u$-algebra such that $A=\Gamma_{f}(V, u)$. We first remark that $\Psi(\sqrt{\Phi(I)})=\sqrt{I}$, using the notation of Remark 2.3. Indeed:

$$
\begin{gathered}
x \in \Psi(\sqrt{\Phi(I)}) \quad \Leftrightarrow \quad x \in \sqrt{\Phi(I)} \cap[0, u] \quad \Leftrightarrow \\
0 \leq x \leq u \text { and } x \in \sqrt{\Phi(I)} \Leftrightarrow
\end{gathered}
$$

$0 \leq x \leq u$ and there exists $n \in \mathbb{N}$ such that $x^{n} \in \Phi(I) \quad \Leftrightarrow$ $0 \leq x \leq u$ and $\left|x^{n}\right| \wedge u \in I \quad \Leftrightarrow \quad x \in A$ and $x^{n} \wedge u=x^{n} \in I \quad \Leftrightarrow \quad x \in \sqrt{I}$. By Remark 2.3, $\Phi(I)$ is an $\ell$-ideal, then $\sqrt{\Phi(I)}$ is an $\ell$-ideal by [14, Proposition 4.2] and again by $\operatorname{Remark}[2.3 \Psi(\sqrt{\Phi(I)})$ is a -ideal.

\section{Classes of $f \mathrm{MV}$-algebras}

In the theory of $f$-algebras, the Archimedean, the semisimple, the semiprime and the formally real structures are proper subclasses studied in the literature. We introduce and briefly investigate the same notions for $f \mathrm{MV}$-algebras. Our main goal is achieved in Section 3.4 where we characterize the quasi-variety of $f \mathrm{MV}$-algebras generated by $[0,1]$.

\subsection{Semisimple and Archimedean $f \mathrm{MV}$-algebras}

It is known that Archimedean MV-algebras and semisimple MV-algebras coincide. The same holds for Riesz MV-algebras, since a Riesz MV-algebra has the same congruences as its MV-algebra reduct. The theory of $f \mathrm{MV}$-algebras has this property no longer.

We recall that an $f$-algebra is Archimedean if $n a \leq b$ for any $n \in \mathbb{N}$ implies $a \leq 0$. The notion of semisimple $f$-algebra is present in literature in different forms, due to the different kind of radicals that can be defined on a $f$-ring (see for example [1, Section 8.6]). We will follow [17], and define an $f$-algebra $\ell$-semisimple if the intersection of all maximal $\ell$-ideals is $\{0\}$. 
Definition 3.1. Let $A$ be an $f \mathrm{MV}$-algebra and let $(V, u)$ be an $f u$-algebra such that $A \simeq \Gamma_{f}(V, u)$. We say that the $f \mathrm{MV}$-algebra $A$ is Archimedean ( $\ell$ semisimple) if the $f u$-algebra $V$ is Archimedean ( $\ell$-semisimple).

We note that an $f \mathrm{MV}$-algebra $A$ is Archimedean if and only if its MV-algebra reduct $\mathcal{U}_{(\mathbb{R}, \cdot)}(A)$ is a semsimple $\mathrm{MV}$-algebra.

Proposition 3.1. Let $A$ be an Archimedean fMV-algebra. Then $A$ is commutative with respect to the internal product.

Proof. Let $(V, u)$ be the $f u$-algebra such that $A \simeq \Gamma_{f}(V, u)$. By definition, $A$ is Archimedean if and only if $(V, u)$ is Archimedean. Therefore, the result follows from [26, Theorem 140.10].

Lemma 3.1. Any $\ell$-semisimple fMV-algebra is Archimedean.

Proof. If we denote by $\ell R a d(A)$ the intersection of all maximal --ideal for the $f \mathrm{MV}$-algebra $A$, since any --ideal is an ideal for the reduct $\mathcal{U}_{(\mathbb{R}, \cdot)}(A)$ it follows that $\operatorname{Rad}(A) \subseteq \ell \operatorname{Rad}(A)$. Then, if $A$ is $\ell$-semisimple, so it is $\mathcal{U}_{(\mathbb{R}, \cdot)}(A)$, and $A$ is Archimedean.

Remark 3.1. If $A$ is a unital $f \mathrm{MV}$-algebra then $A$ is Archimedean if and only if $A$ is $\ell$-semisimple. This result is a direct consequence of Remark 2.2 (2): in this case any ideal is a --ideal.

For unital $f \mathrm{MV}$-algebras, the two classes coincide. Our main interest focuses on this case, but the general relation between Archimedeanity and semisimplicity in the non-unital case will make the subject of future studies.

Proposition 3.2. Let $A_{1}, A_{2}$ be semisimple and unital $f M V$-algebras, and let $f: A_{1} \rightarrow A_{2}$ be a homomorphism of Riesz $M V$-algebras. Then $f$ is also a homomorphism of PMV-algebras.

Proof. By Theorem 2.2 there exist two unital and Archimedean $f$-algebras $\left(V_{1}, u_{1}\right)$ and $\left(V_{2}, u_{2}\right)$ such that $A=\Gamma_{f}\left(V_{1}, u_{1}\right)$ and $B=\Gamma_{f}\left(V_{2}, u_{2}\right)$. The map $f^{\sharp}$ that extend $f$ is a positive map between Archimedean and unital $f$-algebras such that $f^{\sharp}(v)=u$. Therefore by [15, Corollary 5.5], $f^{\sharp}$ is also an algebra homomorphism, and $f: A_{1} \rightarrow A_{2}$ is a homomorphism of PMV-algebras, as well as a homomorphism of Riesz MV-algebras.

\subsection{Semiprime $f \mathrm{MV}$-algebras}

We recall that a semiprime $f$-algebra is an algebra without nilpotents [26, Chapter 142].

Definition 3.2. An element $x$ of an $f \mathrm{MV}$-algebra ( $f$-algebra) is nilpotent if there exists an positive integer $n$ such that $x^{n}=\underbrace{x \cdot \ldots \cdot x}_{n \text { times }}=0$. An $f$ MV-algebra $A$ $(f$-algebra $V)$ is called semiprime if $x^{n}=0$ implies $x=0$. 
Remark 3.2. One can easily see that an $f$ MV-algebra $A$ (f-algebra $V)$ is semiprime if and only if $x \cdot x=0$ implies $x=0$ for any $x \in A(x \in V)$.

Proposition 3.3. Assume $A$ is an $f M V$-algebra and $(V, u)$ is an fu-algebra such that $A \simeq \Gamma_{f}(V, u)$. Then $A$ is semiprime if and only if $(V, u)$ is semiprime.

Proof. In one direction it is obvious. For the other direction, in the case of positive elements the proof is a simple matter of computation; then the result follows from the positive element case by [26. Theorem 142.1(iv)], that is the remark that $x^{+} \cdot x^{-}=0=x^{-} \cdot x^{+}$.

Remark 3.3. Since the property of being semiprime depends only on the underlying $f$-ring structure, a similar result holds for $\operatorname{PMV} f$-algebras and $f$-rings. We state it without proof.

Proposition 3.4. Assume $P$ is a commutative and unital $P M V$-algebra and $(R, u)$ is an fu-ring such that $P \simeq \Gamma_{(\cdot)}(R, u)$. Then $P$ is a $P M V^{+}$-algebra if and only if $(R, u)$ is semiprime.

Corollary 3.1. Let $A$ be a semisimple and unital $f M V$-algebra. Then $A$ is semiprime.

Proof. Let $(V, u)$ be the $f u$-algebra such that $A=\Gamma_{f}(V, u)$. By hypothesis, $A$ is Archimedean, so $V$ is also Archimedean and $u$ is a unit for the product. By [26. Theorem 142.5(iii)] it follows that $(V, u)$ is semiprime, so $A$ is semiprime by Proposition 3.3 .

Corollary 3.2. Let $A$ be a semiprime $f M V$-algebra. Then for any $x, y \in A$, $x^{2}=y^{2}$ if and only if $x=y$.

Proof. The result follows directly by Proposition 3.3 and [26, Theorem 142.3(ii)].

We shall further analyze the commutative, unital and semiprime algebras in the following sections.

\subsection{Formally real $f \mathrm{MV}$-algebras}

While the varieties of Abelian $\ell$-groups and Riesz spaces are generated by $\mathbb{R}$ endowed with the corresponding structure, this is not true for the varieties of $f$-rings and $f$-algebras and counterexamples can be found in [12, 18. Consequently, algebras from $\operatorname{HSP}([0,1])$ were called formally real.

The same facts remain true when applying the $\Gamma$ functors (see Figure 3 ). The varieties of MV-algebras and Riesz MV-algebras are generated by the real interval $[0,1]$ endowed with the corresponding structure (see [6] and, respectively [9]), while the varieties of (commutative and unital) PMV-algebras and $f \mathrm{MV}$-algebras are not. For PMV-algebras, a counterexample was given in [13. This example also stands for $f \mathrm{MV}$-algebras and we briefly recall it in the next example. 
Example 3.1. In [13, Example 3.14] the authors define a totally-ordered finite monoid $S$ and they consider the set $F[S]=\left\{r_{1} X^{s_{1}}+\ldots+r_{n} X^{s_{n}} \mid n \in \mathbb{N}, r_{i} \in\right.$ $F s_{i} \in S$ \}, where $F$ is the ordered field of real numbers. They further identify $X^{\top}$ with 0 , where $\top$ is the greatest element of $S$ and they denote $F[S]_{h}$ the quotient obtained in this way, which is an $f$-ring. Hence the interval $[\mathbf{0}, \mathbf{1}]$ of $F[S]_{h}$ is a PMV-algebra that does not satisfy the following identity:

$\left(x_{1} \cdot z_{1} \ominus y_{1} \cdot z_{2}\right) \wedge\left(x_{2} \cdot z_{2} \ominus y_{2} \cdot z_{1}\right) \wedge\left(y_{1} \cdot y_{2} \ominus x_{1} \cdot x_{2}\right)=0$.

Since the identity holds in the real interval $[0,1]$, it provides the intended countexample in the context of PMV-algebras. We only have to note that $F[S]_{h}$ is in fact an $f$-algebra so $[\mathbf{0 ,}, \mathbf{1}]$ is an $f \mathrm{MV}$-algebra that does not belong to $\operatorname{HSP}([0,1])$.

Definition 3.3. Following [12, we will call an $f \mathrm{MV}$-algebra (PMV-algebra) formally real if it belongs to $\operatorname{HSP}([0,1])$. We denote by $\mathbb{F} \mathbb{R}$ the class of formally real $f \mathrm{MV}$-algebras.

Remark 3.4. In general, a formally real $f$-ring is not unital. For PMV-algebras and $f \mathrm{MV}$-algebras the situation is different: by $[8]$ the unit is the greatest element of the algebras and it belongs to the language. Then the condition for the unit is not existential but universal, that is $1 \cdot x=x \cdot 1=x$ for any $x$.

By well-known results of universal algebra (see for example [11]), the free $f \mathrm{MV}$-algebra in $\mathbb{F} \mathbb{R}$ exists and its elements are term functions defined on $[0,1]$. More precisely, the language of $f \mathrm{MV}$-algebras is

$\mathcal{L}_{f}=\left\{\oplus, \cdot{ }^{*}, 0,\right\} \cup\left\{\delta_{\alpha} \mid \alpha \in[0,1]\right\}$,

where $\delta_{\alpha}$ is a unary operation that is interpreted by $x \mapsto \alpha x$ for any $\alpha \in[0,1]$. For any $n \geq 1$, let $X=\left\{x_{1}, \ldots, x_{n}\right\}$ and assume Term $_{n}$ is the set of $\mathcal{L}_{f}$-terms with variables from $X$. We denote by $F R_{n}$ the free $f \mathrm{MV}$-algebra in $\mathbb{F} \mathbb{R}$ with $n$ free generators. It follows that

$$
F R_{n}=\left\{\widetilde{t} \mid t \in \operatorname{Term}_{n}, \widetilde{t}:[0,1]^{n} \rightarrow[0,1] \text { is the term function of } t\right\} .
$$

In order to characterize $F R_{n}$ we give the following definition.

Definition 3.4. A piecewise polynomial function defined on the $n$-cube is a continuous function $f:[0,1]^{n} \rightarrow[0,1]$ such that there exists a finite number of polynomials $f_{1}, \ldots, f_{k} \in \mathbb{R}\left[x_{1}, \ldots, x_{n}\right]$ with the property that $f\left(a_{1}, \ldots, a_{n}\right)=$ $f_{i}\left(a_{1}, \ldots, a_{n}\right)$ for any $\left(a_{1}, \ldots, a_{n}\right) \in[0,1]^{n}$ and for some $i \in\{1, \ldots, k\}$.

The polynomials $f_{i}$ are called the components of $f$.

Proposition 3.5. The elements of $F R_{n}$ are piecewise polynomial functions defined on the $n$-cube.

Proof. Let $t$ be a term in Term ${ }_{n}$. The result will be proved by structural induction on $t$. We recall that any terms in $\mathrm{Term}_{n}$ is built from the language $\mathcal{L}_{f}$ and the set of variables $\left\{x_{i}\right\}_{i \leq n}$.

If $t=x_{i}$ for some $i \leq n$, then $\widetilde{t}=\pi_{i}^{n}$, the $i^{t h}$ projection and it is trivially a piecewise polynomial function.

If $t=t_{1}^{*}$, then $\widetilde{t}={\widetilde{t_{1}}}^{*}$. By induction hypothesis there exists an integer $h$ and some polynomials $q_{1}, \ldots, q_{h} \in \mathbb{R}\left[x_{1}, \ldots, x_{n}\right]$ such that for any point in the $n$ cube, $\widetilde{t_{1}}$ coincides with one of them. Then $1-q_{1}, \ldots, 1-q_{h}$ are the components of $\widetilde{t}$. 
If $t=t_{1} \oplus t_{2}$, let $q_{1}, \ldots, q_{m}$ be the components of $\widetilde{t_{1}}$ and $p_{1}, \ldots, p_{k}$ be the components of $\widetilde{t_{2}}$. Then $\widetilde{t}$ is defined by the set of polynomials $\{1\} \cup\left\{s_{i j}\right\}_{i, j}$, where $s_{i j}=1-q_{i}+p_{j}$ for any $i \in\{1, \ldots, m\}$ and $j \in\{1, \ldots, k\}$.

If $t=\delta_{\alpha}\left(t_{1}\right)$ for some $\alpha \in[0,1]$ and $q_{1}, \ldots, q_{s}$ are the components of $\widetilde{t_{1}}$, then $\alpha q_{1}, \ldots, \alpha q_{s}$ are the components of $\widetilde{t}$.

If $t=t_{1} \cdot t_{2}$, let $q_{1}, \ldots, q_{m}$ be the components of $\widetilde{t_{1}}$ and $p_{1}, \ldots, p_{k}$ be the components of $\widetilde{t_{2}}$. Then $\widetilde{t}$ is defined by the polynomials $q_{i} \cdot p_{j}$, for any $i \in$ $\{1, \ldots, m\}$ and $j \in\{1, \ldots, k\}$.

The converse of the above proposition is related to the Pierce-Birkhoff conjecture [3, 12] and it will be analyzed in Section 5 .

\section{$3.4 \quad \mathbf{F R}^{+}$-algebras}

The class of $\mathrm{FR}^{+}$-algebras is the quasi-variety of $f \mathrm{MV}$-algebras generated by $[0,1]$. In order to characterize this class, we follow the ideas from [22], where a similar investigation is done for PMV-algebras.

The $\mathrm{FR}^{+}$-algebras are the core of our development, from the algebraic point of view. We prove a representation theorem w.r.t. totally-ordered structures, as well as a representation theorem via ultrapowers of $[0,1]$. In Section 4.2 we develop a logical system that has $\mathrm{FR}^{+}$-algebras as models and in Section 5 we connect them this logic with the Pierce-Birkhoff conjecture.

We recall that a PMV-algebra $A$ is a $P M V^{+}$-algebra [22] if it is unital, commutative and it satisfies the condition:

(sp) $x \cdot x=0$ implies $x=0$ for any $x \in A$.

Theorem 3.1. For an $f M V$-algebra $A$ the following are equivalent:

(1) $A \in \operatorname{ISP}([0,1])$,

(2) $A$ is a unital, commutative and semiprime $f M V$-algebra,

(3) $\mathcal{U}_{\mathbb{R}}(A)$, the $P M V$-algebra reduct of $A$, is a $P M V^{+}$-algebra,

(4) $A$ is in $\mathbb{F} \mathbb{R}$ and $x \cdot x=0$ implies $x=0$ for any $x \in A$.

Proof. (1) $\Leftrightarrow(2)$ One direction is trivial, since any element in $\operatorname{ISP}([0,1])$ is unital, commutative and semiprime. For the other direction, let $\mathbf{V}$ be the class of unital, commutative and semiprime $f \mathrm{MV}$-algebras. If $A \in \mathbf{V}$, then $\mathcal{U}_{\mathbb{R}}(A)$ is a $P M V^{+}$-algebras, and by [22, Corollary 4.4] it belongs to $\operatorname{ISP}([0,1])$, therefore $A$ is a subalgebra of a direct product of copies of $[0,1]$, and the direct product is trivially a $f \mathrm{MV}$-algebra. The map that gives the inclusion is a homomorphism of PMV-algebras, therefore it is a homomorphism of $f \mathrm{MV}$-algebras, and $A$ belongs to $\operatorname{ISP}([0,1])$ as $f$ MV-algebra.

$(2) \Leftrightarrow(3)$ is obvious.

$(1) \Rightarrow(4)$ Since $\operatorname{ISP}([0,1]) \subseteq \operatorname{HSP}([0,1])$, it is straightforward.

$(4) \Rightarrow(2)$ Any formally real $f M V$-algebra is unital and commutative, and the quasi-identity in the hypothesis characterizes semiprime $f \mathrm{MV}$-algebras.

Definition 3.5. Following $[22]$ we denote $\mathbb{F R}^{+}=\operatorname{ISP}([0,1])$, the quasi-variety generated by the standard $f \mathrm{MV}$-algebra $[0,1]$. An $f \mathrm{MV}$-algebra $A$ is a $\mathrm{FR}^{+}$algebra if it is unital, commutative and semiprime. 
Proposition 3.6. $F R_{n}$ belongs to $\mathbb{F R}^{+}$, for any positive integer $n$.

Proof. By Theorem 3.1 we just need to prove that $F R_{n}$ is semiprime. Let $g \in F R_{n}$ such that $g^{2}=\mathbf{0}$, the null function. This means that $g(\mathbf{x}) \cdot g(\mathbf{x})=0$ for every $\mathbf{x} \in[0,1]^{n}$. Since $g(\mathbf{x}) \in[0,1]$, the above condition implies $g(\mathbf{x})=0$ for any $\mathbf{x} \in[0,1]^{n}$, and $g=\mathbf{0}$.

For $\mathrm{FR}^{+}$-algebras we prove the subdirect representation theorem w.r.t. totallyordered $\mathrm{FR}^{+}$-algebras, as well as the representation as an algebra of * $[0,1]$ valued functions.

Theorem 3.2. Any $F R^{+}$-algebra is a subdirect product of totally-ordered $F R^{+}$algebras.

Proof. It is similar to the proof of Theorem 2.1, by [13, Theorem 3.9].

Theorem 3.3. For any $F R^{+}$-algebra $A$ there exists an ultrapower ${ }^{*}[0,1]$ of $[0,1]$ and a set $I$ such that $A$ is embedded in $(*[0,1])^{I}$.

Proof. We first prove the result for totally-ordered $\mathrm{FR}^{+}$-algebras. By hypothesis, the PMV-algebra reduct of $A$ is a $P M V^{+}$-chain, therefore by [22, Corollary 4.4] $A$ can be embedded, as a PMV-algebra, in an ultrapower of $[0,1]$. Since any PMV-algebras homomorphism is also a Riesz MV-algebras homomorphism, the embedding is between $f \mathrm{MV}$-algebras.

By Proposition 3.2 any $\mathrm{FR}^{+}$-algebra $A$ is subdirect product of a family $\left\{A_{i}\right\}_{i}$ of totally-ordered $\mathrm{FR}^{+}$-algebras. We know that any $A_{i}$ is embedded in $U_{i}$, an ultrapower of $[0,1]$. By [5, Proposition 3.1.4 and Corollary 4.3.13] there exists an ultrapower $U$ of $[0,1]$ such that every $U_{i}$ is embedded in $U$. It follows that $A$ is embedded in $\Pi_{i} U$.

\section{Logic for unital and commutative $f \mathrm{MV}$-algebras}

In this section, we define the propositional calculus $\mathcal{F} \mathcal{M V} \mathcal{L}$, that has unital and commutative $f \mathrm{MV}$-algebras as models, as well as the propositional calculus $\mathcal{F} \mathcal{M V L}^{+}$that has $\mathrm{FR}^{+}$-algebras as models. For the latter we prove standard completeness w.r.t. $[0,1]$.

\subsection{The propositional calculus $\mathcal{F} \mathcal{M V \mathcal { L }}$}

The language of the propositional logic $\mathcal{F} \mathcal{M V} \mathcal{L}$ consists of:

(i) a countable set of propositional variables $v_{1}, v_{2}, \ldots$;

(ii) the binary connectives are $\rightarrow$ and ';

(iii) the unary connective $\neg$;

(iv) a family of unary connectives $\left\{\nabla_{\alpha} \mid \alpha \in[0,1]\right\}$;

(v) the parentheses ( and).

Formulas, theorems, deductions, proof are defined as usual. The axioms for the logic of unital and commutative $f \mathrm{MV}$-algebras will be the following: 
(L1) $\varphi \rightarrow(\psi \rightarrow \varphi)$

(L2) $(\varphi \rightarrow \psi) \rightarrow((\psi \rightarrow \chi) \rightarrow(\varphi \rightarrow \chi))$

(L3) $(\varphi \vee \psi) \rightarrow(\psi \vee \varphi)$

(L4) $(\neg \psi \rightarrow \neg \varphi) \rightarrow(\varphi \rightarrow \psi)$

(R1) $\nabla_{\alpha}(\varphi \rightarrow \psi) \leftrightarrow\left(\nabla_{\alpha} \varphi \rightarrow \nabla_{\alpha} \psi\right)$

(R2) $\nabla_{\left(\alpha \odot \beta^{*}\right)} \varphi \leftrightarrow\left(\nabla_{\beta} \varphi \rightarrow \nabla_{\alpha} \varphi\right)$

(R3) $\nabla_{\alpha}\left(\nabla_{\beta} \varphi\right) \leftrightarrow \nabla_{\alpha \cdot \beta} \varphi$

(R4) $\nabla_{1} \varphi \leftrightarrow \varphi$

(P1) $(\chi \cdot(\varphi \ominus \psi)) \leftrightarrow((\chi \cdot \varphi) \ominus(\chi \cdot \psi))$

(P2) $(\varphi \cdot(\psi \cdot \chi)) \leftrightarrow((\varphi \cdot \psi) \cdot \chi)$

(P3) $\varphi \rightarrow(\varphi \cdot(\varphi \rightarrow \varphi))$

(P4) $\varphi \cdot \psi \rightarrow \varphi$

(P5) $\varphi \cdot \psi \leftrightarrow \psi \cdot \varphi$

(A1) $\Delta_{\alpha}(\varphi \cdot \psi) \leftrightarrow\left(\Delta_{\alpha} \varphi \cdot \psi\right)$

(A2) $\Delta_{\alpha}(\varphi \cdot \psi) \leftrightarrow\left(\varphi \cdot \Delta_{\alpha} \psi\right)$,

where $\varphi \ominus \psi$ means $\neg(\varphi \rightarrow \psi), \Delta_{\alpha} \varphi$ means $\neg \nabla_{\alpha}(\neg \varphi)$ and the only deduction

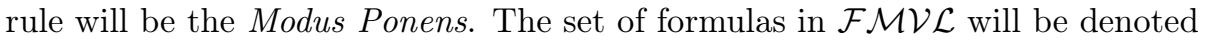
by $F_{f M V}$.

Note that (L1)-(L4) are the axioms of Łukasiewicz logic.

For a subset $\Theta \subseteq F_{f M V}$ we define an equivalence relation as follows:

$$
\varphi \equiv_{\Theta} \psi \quad \text { iff } \quad \Theta \vdash \varphi \rightarrow \psi \text { and } \Theta \vdash \psi \rightarrow \varphi \text {. }
$$

We define the following operation on $F_{f M V} / \equiv_{\Theta}$ :

- $[\varphi]_{\Theta}^{*}=[\neg \varphi]_{\Theta}, \quad[\varphi]_{\Theta} \rightarrow[\psi]_{\Theta}=[\varphi \rightarrow \psi]_{\Theta}$

- $[\varphi]_{\Theta} \oplus[\psi]_{\Theta}=[\neg \varphi \rightarrow \psi]_{\Theta}, \quad[\varphi]_{\Theta} \cdot[\psi]_{\Theta}=[\varphi \cdot \psi]_{\Theta}$;

- $\alpha[\varphi]_{\Theta}=\left[\Delta_{\alpha} \varphi\right]_{\Theta}$

- $1_{\Theta}=T_{\mathcal{F} \mathcal{M V} \mathcal{L}}(\Theta), \quad 0_{\Theta}=1_{\Theta}^{*}$.

Theorem 4.1. The structure $f M V L(\Theta)=\left(\left(F_{f M V} / \equiv_{\Theta}, \oplus, *, \cdot, 0_{\Theta}\right)\right.$, $\left.\Phi\right)$, where $\Phi:[0,1] \times F_{f M V} \rightarrow F_{f M V}, \Phi(\alpha,[\varphi])=\left[\Delta_{\alpha} \varphi\right]$, is a unital and commutative $f M V$-algebra.

Proof. By [9, Proposition 5], this structure is a Riesz MV-algebra. By [13, Lemma 3.21] it is a unital and commutative PMV-algebra, and by axioms (A1) and (A2) we get the algebra's relations between internal product and scalar product. Trivially any unital PMV-algebra is an PMVf-algebra.

In the sequel, we will denote by $f M V L$ the Lindenbaum-Tarski algebra of the logic, that is $f M V L(\Theta)$ with $\Theta=\emptyset$.

An appropriate semantic for the logic has unital and commutative $f \mathrm{MV}$ algebras as models. Let $A$ be an $f \mathrm{MV}$-algebra; an evaluation is a function $e: F_{f M V} \rightarrow A$ such that:

(e1) $e(\varphi \rightarrow \psi)=e(\varphi)^{*} \oplus e(\psi)$

(e2) $e\left(\neg \varphi=e(\varphi)^{*}\right)$

(e3) $e\left(\nabla_{\alpha} \varphi\right)=\left(\alpha e(\varphi)^{*}\right)^{*}$

(e4) $e(\varphi \cdot \psi)=e(\varphi) \cdot e(\psi)$

for any formulas $\varphi$ and $\psi$ of $\mathcal{F} \mathcal{M V} \mathcal{L}$.

We have the following completeness theorem. 
Theorem 4.2. Let $\Theta$ be a set of formulas and $\varphi$ a formula in $F_{f M V}$. The following are equivalent:

(1) $\Theta \vdash \varphi$,

(2) $\Theta \models_{A} \varphi$ for any fMV-algebra $A$,

(3) $\Theta \models_{A} \varphi$ for any totally-ordered fMV-algebra $A$,

(4) $[\varphi]_{\Theta}=1_{\Theta}$ in $f M V L(\Theta)$.

Proof. (2) $\Leftrightarrow$ (3) follows by Theorem 2.1. The rest of the proof is straightforward.

Proposition 4.1. $\mathcal{F} \mathcal{M V} \mathcal{L}$ is a conservative extension of $\mathcal{L}$, the infinite valued Eukasiewicz logic.

Proof. Let $\varphi$ be a formula in Łukasiewicz logic which is a theorem in $\mathcal{F} \mathcal{M V} \mathcal{L}$. Then by completeness, $\varphi$ is a tautology in the standard $f \mathrm{MV}$-algebra $[0,1]$, and since $\varphi$ does not involves $\cdot$ and $\nabla_{\alpha}$, it is a tautology in the standard MV-algebra $[0,1]$. Then by the completeness of Łukasiewicz logic, $\varphi$ is a theorem in $\mathcal{L}$.

As for Łukasiewicz logic, the deduction theorem holds in its local form.

Remark 4.1. Let $\Theta$ be a non-empty subset of $F_{f M V}$, and $\varphi \in F_{f M V}$. For any $\psi \in F_{f M V}$ we have

$$
\Theta \cup\{\varphi\} \vdash \psi \quad \text { iff } \quad \Theta \vdash \varphi \rightarrow \cdots(\varphi \rightarrow \psi),
$$

where $\varphi$ appears $n$ times for some $n \geq 1$. The proof is similar with the one for Łukasiewicz logic [6, Proposition 4.6.4].

\subsection{The propositional calculus $\mathcal{F} \mathcal{M V \mathcal { L }} \mathcal{L}^{+}$}

The propositional calculus $\mathcal{F} \mathcal{M V} \mathcal{L}^{+}$is obtained from $\mathcal{F} \mathcal{M V L}$ by adding the deduction rule

$$
\text { Semiprime: } \frac{\neg(\varphi \cdot \varphi)}{\neg \varphi} \text {. }
$$

Theorem 4.3. Let $\Theta$ be a set of formulas in $\mathcal{F} \mathcal{M V \mathcal { L } ^ { + }}$ and $\varphi$ a formula. Then the following are equivalent:

(1) $\Theta \vdash \varphi$,

(2) $e(\varphi)=1$ for any $e[0,1]$-model of $\Theta$,

(3) $e(\varphi)=1_{A}$ for any algebra $A$ from $\mathbb{F}^{+}$and for any e A-model of $\Theta$.

Proof. (1) $\Rightarrow(2)$ It is straightforward, since Modus Ponens and Semiprime preserve tautologies.

$(2) \Rightarrow(3)$ It follows by Theorem 3.1

$(3) \Rightarrow(1)$ Since (3) holds for the algebras from $\mathbb{F R}^{+}$, then (1) follows directly by the definition of $1_{\Theta}$.

It follows that the models of $\mathcal{F} \mathcal{M V \mathcal { L } ^ { + }}$ are $F R^{+}$-algebras. Note that our system is an extension of the system $P L^{\prime}$, defined in [13], that has $\mathrm{PMV}^{+}$algebras as models. 
Proposition 4.2. $\mathcal{F} \mathcal{M V \mathcal { L } ^ { + }}$ is a conservative extension of $\mathcal{L}$.

Proof. It is similar to the proof for $\mathcal{F} \mathcal{M V} \mathcal{L}$.

Remark 4.2. $\mathcal{F} \mathcal{M V} \mathcal{L}^{+}$does not satisfy the deduction theorem in the same form of $\mathcal{F} \mathcal{M V} \mathcal{L}$ : the counterexample in [13, Corollary 3.19] applies.

The appropriate semantic for $\mathcal{F} \mathcal{M V} \mathcal{L}^{+}$is the one with unital, commutative and semiprime $f \mathrm{MV}$-algebras, that is, algebras in $\mathbb{F R}^{+}$, as models with the usual definition for evaluations.

Remark 4.3. Let $n \geq 1$ be a natural number. The Linbenbaum-Tarski algebra with $n$ variables, denoted by $f M V L_{n}^{+}$, is isomorphic with the free $\mathrm{FR}^{+}$-algebra with $n$ free variables. By Propositions 3.5] and 3.6, we infer that $f M V L_{n}^{+} \simeq F R_{n}$ and we know that the elements of $F R_{n}$ are $[0,1]$-valued piecewise polynomial functions defined on $[0,1]^{n}$.

In the following section we shall investigate the answer to the following question: does $F R_{n}$ contain all the [0,1]-valued piecewise polynomial functions defined on $[0,1]^{n}$ ?

The following result is a preliminary step.

Proposition 4.3. If $p:[0,1]^{n} \rightarrow \mathbb{R}$ is a polynomial with real coefficients then there exists a formula $\varphi$ with $n$ variables of $\mathcal{F} \mathcal{M V \mathcal { L }}{ }^{+}$such that $((p \vee \mathbf{0}) \wedge \mathbf{1})$ coincides with the term function associated to $\varphi$.

Proof. The proof is similar with the one of [9, Proposition 7.6]. In the following we denote the function $p \mapsto((p \vee \mathbf{0}) \wedge \mathbf{1})$ by $\varrho$, and by $\widetilde{\varphi}$ the term function associated to the formula $\varphi$. Let $p:[0,1]^{n} \rightarrow \mathbb{R}$ be a polynomial function. Let $k$ be the degree of $p$. Then it follows

$$
p\left(x_{1}, \ldots, x_{n}\right)=\sum_{i_{1}+\ldots+i_{n} \leq k} c_{i_{1}, \ldots, i_{n}} x_{1}^{i_{1}} \cdots x_{n}^{i_{n}},
$$

where $c_{i_{1}, \ldots, i_{n}} \in \mathbb{R}$ for any choice of the indexes. We notice that any $c_{i_{1}, \ldots, i_{n}}$ can be written as a sum of a finite number of elements in $[-1,1]$, then we assume that

$$
p\left(x_{1}, \ldots, x_{n}\right)=r_{m} y_{m}+\cdots r_{p+1} y_{p+1}+r_{p}+\cdots+r_{1}
$$

where $m \geq 1$ and $p \geq 0$ are natural numbers, $p \leq m, r_{j} \in[-1,1] \backslash\{0\}$ for any $j \in\{1, \ldots, m\}$ and $y_{j} \in\left\{x_{1}^{i_{1}} \cdots x_{n}^{i_{n}} \mid i_{1}+\ldots+i_{n} \leq k\right\}$ for any $j \in\{p+1, \ldots, m\}$.

We prove the theorem by induction on $m \geq 1$. In the sequel we denote by $\mathbf{x}$ an element $\left(x_{1}, \cdots, x_{n}\right)$ from $[0,1]^{n}$.

Initial step $m=1$. We have $p(\mathbf{x})=r$ for any $\mathbf{x} \in[0,1]^{n}$ or $p(\mathbf{x})=r x_{1}^{i_{1}} \cdots x_{n}^{i_{n}}$ for any $\mathbf{x} \in[0,1]^{n}$ where $r \in[-1,1] \backslash\{0\}$ and $\left\{i_{1}, \ldots, i_{n}\right\}$ is a suitable set of index.

If $r \in[-1,0)$ then $\varrho(p)=0$ so $\varrho \circ p=\widetilde{\varphi}$ for $\varphi=\overline{0}$.

If $r \in(0,1]$ then $p=\varrho \circ p$. It follows that $p=\widetilde{\varphi}$ where $\varphi=\delta_{r}\left(\overline{0}^{*}\right)$ if $p(\mathbf{x})=r$ for any $\mathbf{x} \in[0,1]^{n}$, and $\varphi=\delta_{r}\left(v_{1}^{i_{1}} \cdot \ldots \cdot v_{n}^{i_{n}}\right)$ if $p(\mathbf{x})=r x_{1}^{i_{1}} \cdots x_{n}^{i_{n}}$ for 
any $\mathbf{x} \in[0,1]^{n}$.

Induction step. We take $p=g+h$ where $\varrho \circ g=\widetilde{\varphi_{1}}$ for some formula $\varphi_{1}$ and there is $r \in[-1,1] \backslash\{0\}$ and a suitable choice of index for $i_{1}, \ldots, i_{n}$ such that $h(\mathbf{x})=r$ for any $\mathbf{x} \in[0,1]^{n}$, or $h(\mathbf{x})=r x_{1}^{i_{1}} \cdots x_{n}^{i_{n}}$ for any $\mathbf{x} \in[0,1]^{n}$. We consider two cases.

Case 1. If $r \in(0,1]$ then $h:[0,1]^{n} \rightarrow[0,1]$ so $\varrho \circ p=((\varrho \circ g) \oplus h) \odot(\varrho \circ(1+g))$ by [9, Lemma 10]. Following the initial step, there is a formula $\varphi_{2}$ such that $h=\widetilde{\varphi_{2}}$. We notice that $1+g=1-(-g)$ and the induction hypothesis holds for $(-g)$, then there is a formula $\varphi_{3}$ such that $\varrho \circ(-g)=\widetilde{\varphi_{3}}$. It follows by [9, Lemma 10], $\varrho \circ(1+g)=1-\widetilde{\varphi_{3}}={\widetilde{\varphi_{3}}}^{*}$. We get $\varrho \circ p=\widetilde{\varphi}$ where $\varphi=\left(\varphi_{1} \oplus \varphi_{2}\right) \odot \varphi_{3}^{*}$. Case 2. If $r \in[-1,0)$, then $g+h=(g-1)+(1+h)$ and $1+h:[0,1]^{n} \rightarrow[0,1]$. By [9, Lemma 10] we get

$$
\varrho \circ p=((\varrho \circ(g-1)) \oplus(1+h)) \odot(\varrho \circ g) .
$$

Following the initial step, there is a formula $\varphi_{2}$ such that $-h=\widetilde{\varphi_{2}}$, so $1+h=$ $1-(-h)={\widetilde{\varphi_{2}}}^{*}$. In the sequel we have to find a formula $\varphi_{3}$ that corresponds to $\varrho \circ(g-1)$, where

$$
g(\mathbf{x})=r_{m} y_{m}+\cdots+r_{p+1} y_{p+1}+r_{p}+\cdots+r_{1}
$$

with $r_{j} \in[-1,1] \backslash\{0\}$ for any $j \in\{1, \ldots, m\}$ and $y_{j}$ in $\left\{x_{1}^{i_{1}} \cdots x_{n}^{i_{n}} \mid i_{1}+\ldots+i_{n} \leq\right.$ $k\}$ for any $j \in\{p+1, \ldots, m\}$.

Case 2.1. If $r_{j} \leq 0$ for any $j \in\{1, \ldots, m\}$ then $g-1 \leq 0$, so $\varrho \circ(g-1)=0=\widetilde{\varphi_{3}}$ with $\varphi_{3}=0$.

Case 2.2. If there is $j_{0} \in\{1, \ldots, p\}$ such that $r_{j_{0}}>0$, then

$$
(g-1)(\mathbf{x})=r_{m} y_{m}+\cdots+r_{p+1} y_{p+1}+r_{p}+\cdots+\left(r_{j_{0}}-1\right)+\cdots+r_{1}
$$

and $r_{j_{0}}-1 \in[-1,0)$, so the induction hypothesis applies to $g-1$. Then there exists a formula $\varphi_{3}$ such that $\varrho \circ(g-1)=\widetilde{\varphi_{3}}$.

Case 2.3. If there is $j_{0} \in\{p+1, \cdots, m\}$ such that $r_{j_{0}}>0$, then we set $h_{0}(\mathbf{x})=r_{j_{0}} y_{j_{0}}$ and

$$
g_{0}(\mathbf{x})=g(\mathbf{x})-r_{j_{0}} y_{j_{0}}-1 .
$$

It follows that $g-1=g_{0}+h_{0}$ such that $g_{0}$ satisfies the induction hypothesis and $h_{0}:[0,1]^{n} \rightarrow[0,1]$. We are in the hypothesis of Case 1 , so there exists a formula $\varphi_{3}$ such that $\varrho \circ(g-1)=\widetilde{\varphi_{3}}$.

Summing up, we get $\varrho \circ(g+h)=\widetilde{\varphi}$ with $t=\left(\left(\varphi_{2} \oplus \varphi_{3}^{*}\right) \odot \varphi_{1}\right)$.

\section{Connections with the Pierce-Birkhoff conjec- ture}

At the end of the paper [3], the authors asked for a characterization of the "free,

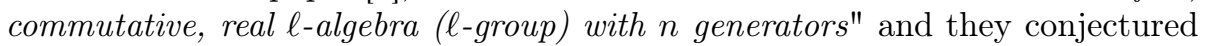
that "it is isomorphic with the l-group of real functions which are continuous and piecewise polynomial of degree at most n over a finite number of pieces". They asked "the same problem for the free (commutative) $\ell$-rings, for free $f$-rings", saying that: "The former is probably very difficult".

Definition 5.1. Let $n \geq 1$ be a natural number. 
- A function $f: \mathbb{R}^{n} \rightarrow \mathbb{R}$ is a piecewise polynomial (PWP) function if it is continuous and there is a finite set of polynomials $\left\{p_{1}, \ldots, p_{k}\right\} \in$ $\mathbb{R}\left[x_{1}, \ldots, x_{n}\right]$ such that for any $\left(a_{1}, \ldots, a_{n}\right) \in \mathbb{R}^{n}$ there exists $i \in\{1, \ldots, k\}$ with $f\left(a_{1}, \ldots, a_{n}\right)=p_{i}\left(a_{1}, \ldots, a_{n}\right)$.

- A continuous function $f: \mathbb{R}^{n} \rightarrow \mathbb{R}$ is a inf-sup-polynomial-definable (ISD) function if there is a finite set of polynomials $\left\{q_{i j} \mid 1 \leq i \leq m, 1 \leq j \leq\right.$ $k\} \subseteq \mathbb{R}\left[x_{1}, \ldots, x_{n}\right]$ such that $f=\bigvee_{i=1}^{m} \bigwedge_{j=1}^{k} q_{i j}$.

We denote by $P W P(n)$ the set of all PWP-functions and by $I S D(n)$ the set of all ISD-functions defined as above.

Remark 5.1. The Pierce-Birkhoff conjecture states that $P W P(n)=I S D(n)$ for any $n \geq 2$ and, in this form, it was formulated by Henriksen and Isbell. The proof for $n \leq 2$ was made by L. Mahé in [19, where an unpublished proof of Gus Efroymson is also quoted.

Definition 5.2. Let $n \geq 1$ be a natural number.

- A function $f:[0,1]^{n} \rightarrow[0,1]$ is a $\mathrm{PWP}_{u}$-function if it is continuous and there is a finite set of polynomials with real coefficients $p_{1}, \ldots, p_{k}: \mathbb{R}^{n} \rightarrow$ $\mathbb{R}$ such that for any $\left(a_{1}, \ldots, a_{n}\right) \in \mathbb{R}^{n}$ there exists $i \in\{1, \ldots, k\}$ with $f\left(a_{1}, \ldots, a_{n}\right)=p_{i}\left(a_{1}, \ldots, a_{n}\right)$.

- A continuous function $f:[0,1]^{n} \rightarrow[0,1]$ is an $\operatorname{ISD}_{u}$-function if there is a finite set of polynomials with real coefficients $\left\{q_{i j}:[0,1]^{n} \rightarrow \mathbb{R} \mid 1 \leq i \leq\right.$ $m, 1 \leq j \leq k\}$ such that $f=\bigvee_{i=1}^{m} \bigwedge_{j=1}^{k}\left(\left(q_{i j} \vee \mathbf{0}\right) \wedge \mathbf{1}\right)$.

We denote by $P W P(n)_{u}$ the set of all $\mathrm{PWP}_{u}$-functions and by $I S D(n)_{u}$ the set of all $\mathrm{ISD}_{u}$-functions.

Theorem 5.1. The following properties hold:

(1) $I S D(n)_{u} \subseteq F R_{n} \subseteq P W P(n)_{u}$ for any $n \in \mathbb{N}$,

(2) $I S D(n)_{u}=F R_{n}=P W P(n)_{u}$, for $n \leq 2$.

Proof. (1) It is a direct consequence of Propositions 4.3 and 3.5 .

(2) Let $f \in P W P(n)_{u}$ with $n \leq 2 . f$ can be extended to a function $\bar{f}: \mathbb{R}^{n} \rightarrow \mathbb{R}$ in the following way: for $n=1$, we set $\bar{f}(x)=c_{0}$ for any $x \leq 0$ and $\bar{f}(x)=c_{1}$ for any $x \geq 1$, where $c_{0}=f(0)$ and $c_{1}=f(1)$; for $n=2$, since $f$ is defined over $[0,1]^{2}$, the result follows by [10, Theorem 1.2]. Therefore, by [19] there exist two set of indexes $I, J$ such that

$$
\bar{f}=\bigvee_{i \in I} \bigwedge_{j \in J} \overline{f_{i j}}
$$

where $\overline{f_{i j}}$ are polynomial functions from $\mathbb{R}^{n}$ to $\mathbb{R}$ with real coefficient. We consider the restrictions $f$ and $f_{i j}$ on $[0,1]^{n}$ of $\bar{f}$ and $\overline{f_{i j}}$ respectively, and we get

$$
f=\varrho \circ f=\bigvee_{i \in I} \bigwedge_{j \in J}\left(\varrho \circ f_{i j}\right)=\bigvee_{i \in I} \bigwedge_{j \in J} \widetilde{\varphi_{i j}},
$$


for some suitable formulas, by Proposition 4.3. We set $\varphi=\bigvee_{i \in I} \bigwedge_{j \in J} \varphi_{i j}$, and we get $f=\widetilde{\varphi}$. Then $P W P(n)_{u}=F R_{n}=I S D(n)_{u}$, for $n \leq 2$.

Remark 5.2. We worked in the context of $f \mathrm{MV}$-algebras, so the components of the piecewise polynomial functions have real coefficients. A similar approach can be used in the context of PMV-algebras, but in this case the components of the piecewise polynomial functions will have integer coefficients. One can easily see that Propositions 4.3, 3.5 and Theorem 5.1 can be proved in a similar way.

Conjecture 5.1. $I S D(n)_{u}=F R_{n}=P W P(n)_{u}$ for any $n \geq 3$.

Conclusion. The Pierce-Birkhoff conjecture implies Conjecture 5.1 in the presence of additional extension results (see [10] for $n=2$ ). Assuming Conjecture 5.1 holds, in order to prove the Pierce-Birkhoff conjecture, one needs results of the following type:

if $f \in P W P(n)$ such that $\left.(f \wedge 1 \vee 0)\right|_{[0,1]^{n}} \in I S D(n)_{u}$ then $f \in I S D(n)$.

Conjecture 5.1 is a normal form theorem for the logical system $\mathcal{F} \mathcal{M V} \mathcal{L}^{+}$, obtained by extending Łukasiewicz logic with both an internal and external product and it can be seen as a local version of the Pierce-Birkhoff conjecture.

\section{Acknowledgements}

Part of this research was carried on while S. Lapenta was visiting University of Bucharest, supported by the grant of the International Doctoral Seminar School Entitled "Janos Bolyai". Both authors wish to thank Professor Antonio Di Nola for encouraging their collaboration, for his comments and discussions on various subjects that, all together, led to the present development.

\section{References}

[1] Bigard A., Keimel K., Wolfstein S., Groupes et Anneuax Reticules, Lecture Notes in Mathematics Springer-Verlag 1977.

[2] Birkhoff G., Lattice Theory, AMS Coll. Publ. 25 3rd Ed. 1973.

[3] Birkhoff G., Pierce R.S., Lattice-ordered Rings, An. Acad. Brasil. Cienc. 28 (1956) 41-69.

[4] Chang C.C., Algebraic Analysis of Many Valued Logics, Trans. Amer. Math. Soc. 88 (1958) 467-490.

[5] Chang C.C., Keisler H.J., Model Theory, North-Holland Amsterdam 1973.

[6] Cignoli R., D’Ottaviano I.M.L., Mundici D., Algebraic Foundation of Many Valued Reasoning, Kluver Academc Publ Dordrecht 2000.

[7] Di Nola A., Representation and Reticulation by Quotients of MV-algebras, Ricerche di Matematica 40(2) (1991) 291-297. 
[8] Di Nola A., Dvurečenskij A., Product $M V$-algebras, Multiple-Valued Logics 6 (2001) 193-215.

[9] Di Nola A., Leustean I., Eukasiewicz Logic and Riesz Spaces, doi: 10.1007/s00500-01k4-1348-z, Soft Computing, 2014.

[10] Fischer A., Marshall M., Extending Piecewise Polynomial Functions in two Variables, Annales de la Faculte des Sciences Toulouse 22 (2013) 253-268.

[11] Grätzer G., Universal Algebra, Springer-Verlag Berlin- Heilderberg-New York 1968.

[12] Henriksen M., Isbell J.R., Lattice-ordered Rings and function Rings, Pacif. J. Math. 12(2) (1962) 533-565.

[13] Horčík R., Cintula P., Product Lukasiewicz logic, Archive for Mathematical Logic 43(4) (2004) 477-503.

[14] Huijsmans C.B., de Pagter B., Ideal Theory in f-algebras, Trans. A.M.S. $269(1)(1982) 225-245$.

[15] Huijsmans C.B., de Pagter B., Subalgebras and Riesz Subspaces in an falgebra, Proc. London Math. Soc. 48(3) (1984) 161-174.

[16] Luxemburg W.A.J., Zaanen A.C., Riesz Spaces I, North-Holland Amsterdam 1971.

[17] Ma J., Wojciechowski P., Structure Spaces of Maximal $\ell$-Ideals of LatticeOrdered Rings, Ordered Algebraic Structures 7 (2002) 261-274.

[18] Madden J., On f-rings that are not formally real, Annales de la faculté des Sciences de Toulouse Mathématiques Volume: 19, Issue: S1 (2010) 143-157.

[19] Mahé L., On the Pierce-Birkhoff conjecture, Rocky M. J. 14(4) (1984) 983985.

[20] McNaughton R., A theorem about Infinite-Valued Sentential Logic, Journal of Symbolic Logic 16 (1951) 1-13.

[21] Montagna F., An algebraic approach to Propositional Fuzzy Logic, Journal of Logic, Language and Information 9 (2000) 91-124.

[22] Montagna F., Subreducts of MV-algebras with product and product residuation, Algebra Universalis 53 (2005) 109-137.

[23] Montagna F., Panti G., Adding structure to MV-algebras, J. Pure and Applied Algebra 164 (2001) 365-387.

[24] Mundici D., Interpretation of ACF ${ }^{*}$-algebras in Eukasiewicz sentential Calculus, J. Funct. Anal. 65 (1986) 15-63. 
[25] Mundici D., Advanced Lukasiewicz calculus and MV-algebras, Trends in Logic 35, Springer, 2011.

[26] Zaneen A.C., Riesz Space II, North Holland, Amsterdam 1983. 